\title{
Evaluation of the Spectroscopic Performance of the Integrated Multi-Channel Charge-Sensitive Preamplifier of TRACE with a Silicon Detector Prototype
}

\author{
Stefano Capra, Ramon J. Aliaga, Daniele Mengoni, Philipp R. John, Andres Gadea, Vicente Herrero, \\ Alberto Pullia.
}

\begin{abstract}
In this work the experimental results are presented showing the spectroscopic performance of the ASIC multichannel charge-sensitive preamplifier of TRACE (TRacking Array for light Charged particle Ejectiles). The results were obtained connecting a silicon pad detector to a custom-designed preamplifier board with eight ASIC CSPs. The detector and the board were put in a vacuum chamber with a triple $\mathrm{Am}-\mathrm{Cm}-\mathrm{Pu}$ alpha source. The output signals were digitized with four FPGApowered $100 \mathrm{MHz}$ 14-bit resolution digitizer cards. The energy resolution obtained is around $22 \mathrm{keV}$ at $5486 \mathrm{keV}$. The results are very encouraging and pave the way for future developments.
\end{abstract}

Index Terms-Charge-Sensitive amplifier, Analog integrated circuits, Spectroscopy, Particle Detectors, Pulse Analysis, LowNoise electronics.

\section{INTRODUCTION}

$\mathbf{I}$ $\mathrm{N}$ the forthcoming experiments with radioactive nuclear beams that will take place in the new facilities under construction such as SPES (Legnaro National Laboratories), SPIRAL2 (Ganil) and FAIR (GSI) nuclear structures far from the valley of stability will be studied. In these experiments the expected counting rate and dynamic range are very high. New highly-segmented detectors will both provide enhanced spatial resolution and lower per-channel count rate. With 100 or less channels it's still possible to hard wire each one to the DAQ infrastructure, but with $1 \mathrm{k}$ or more this is practically not feasible. For these reasons the front-end electronics must employ non-conventional solutions.

The TRACE detector array [1] in its final configuration will consist of several thousands of channels. Both the detectors and the front-end electronics will be placed inside the small volume of the reaction chamber and the connections to the outer world should be minimized.

The solution proposed is to use ASIC preamplifiers connected in groups of 32 channels to analog memory chips [2], [3]. The sampled signals will be multiplexed, packed with

S. Capra is with the Physics Department of University of Milano and INFN of Milano, Italy (email: stefano.capra@unimi.it).

R. J. Aliaga is with the Instituto de Fisica Corpuscular (CSIC-UV), Paterna, Valencia, Spain.

D. Mengoni is with the Department of Physics and Astronomy, University of Padova, Padova, Italy and INFN, Padova, Italy.

A. Gadea is with the Instituto de Fisica Corpuscular (CSIC-UV), Paterna, Valencia, Spain.

V. Herrero is with the Universitat Politecnica de Valencia, Valencia, Spain

A. Pullia is with the Department of Physics, University of Milano, Italy, and INFN of Milano, Italy (email: alberto.pullia@mi.infn.it).

Manuscript received November 15, 2016;

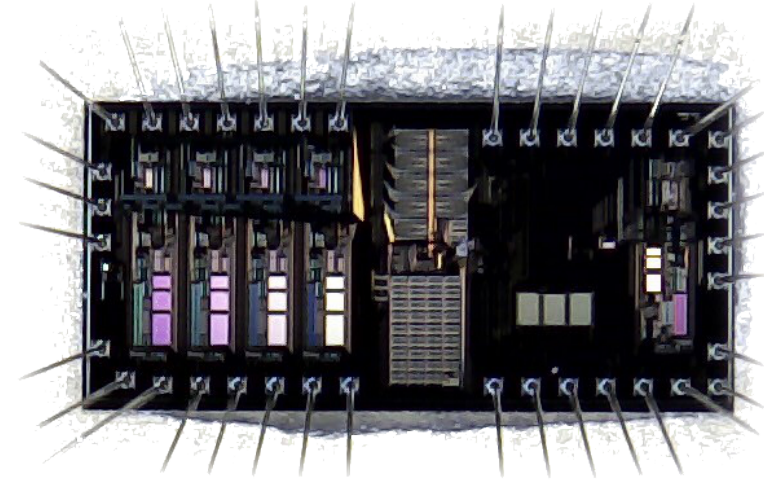

Fig. 1. This photograph of the preamplifier chip was taken with a microscope attached to a PC through USB connection. The total area of the chip is $5 \mathrm{~mm}^{2}$.

timestamp and channel number information and transmitted to the back-end. The digitized signals will be processed with pulse-shape algorithms (PSA) to perform particle discrimination.

\section{The Multi-Channel Integrated Charge-SEnsitive PREAMPLIFIER}

A multi-channel integrated charge-sensitive preamplifier was realized and tested [4], [5]. It was designed in AMS $0.35 \mu \mathrm{m}$ CMOS technology and is intended to be powered with a dual $\pm 2.5 \mathrm{~V}$ supply. It comprises four channel specifically designed for hole signals and one for electron signals. The former are intended to be connected to the front pads of the detectors and the latter to the back electrode through proper decoupling capacitor. All the channels implement a differential input stage and a low-impedance output stage. The first one ensures a $0 \mathrm{~V}$ bias point and the second one is able to drive a $50 \Omega$ terminated cable. The only external component is a $1 \mathrm{G} \Omega$ feedback resistor for each channel. The preamplifier resolution, evaluated on the test-bench with a pulser, is $1.1 \mathrm{keV}$ in silicon, considering $5 \mathrm{pF}$ of detector capacitance. The high bandwidth ensures a signal rise-time around 10 ns. A simple $\mathrm{I}^{2} \mathrm{C}$ interface enables to adjust some key parameters such as gain and bandwidth via slow control. Since the TRACE front-end electronics must be operated in vacuum the power consumption should be kept as low as possible. As the reader surely knows power and performance (both noise and speed) are in trade-off. In this version the ASIC power consumption 


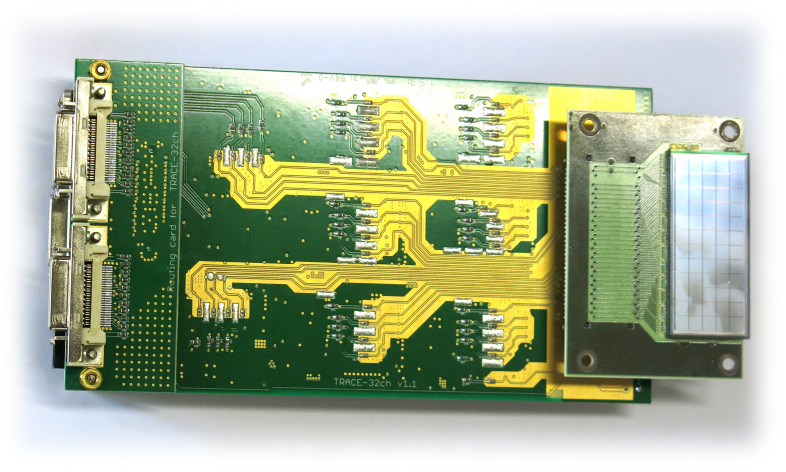

Fig. 2. TRACE32ch v1.1 board connected to a TRACE silicon PAD detector.

is $12 \mathrm{~mW}$ per channel. The electron channel is separately powered and can be shut off when not used.

One of the key features of this integrated preamplifier is an auxiliary fast-reset device [6]-[13]. This device has two main purposes. The first one is to reduce dramatically the deadtime in case of saturation. The time required for a complete recover is around $10 \mu \mathrm{s} / \mathrm{MeV}$. The second purpose is to extend the dynamic range of the preamplifier thanks to a timeover-threshold (TOT) technique. In this way, if the preamplifier is able to process linearly events up to $40 \mathrm{MeV}$ in silicon, with the TOT method the spectroscopic energy range is boosted up to more than $700 \mathrm{MeV}$.

\section{EXPERIMENTAL SETUP AND RESULTS}

In order to connect the integrated charge-sensitive preamplifiers to the TRACE silicon detector prototypes a custom board was designed and realized. This board can host eight chips for a total of 32 front channels. The electron channel is active only on one of these chips and is connected to the back electrode of the detector. The detector is segmented into 60 square, $4 \mathrm{~mm}$ wide pads. Due to the limited number of the readout channels available only the central pads are active. The board is realized with a Rogers $4003 c$ laminate, characterised by low dielectric loss and is compliant with standard FR4 production processes. The output signals are routed to three MDR26 connectors. Proper adapters were used to connect the board to the array of $3 M$ headers on the flange of the vacuum chamber. The output signals were sent to four N1728A CAEN modules. They are FPGA-powered $100 \mathrm{MHz}$ 14-bit resolution digitizer cards with four differential inputs each. A simple digital trigger on the back signal was provided by the digitizer card itself. A triple ${ }^{241} \mathrm{Am}^{2}{ }^{244} \mathrm{Cm}^{239} \mathrm{Pu}$ alpha source was put inside the vacuum chamber. The system was operated at 0.2 mbar. The energy resolution at different energies is reported in table I.

\section{CONCLUSiOnS}

These results, although very promising, should be considered preliminary. This result was due not only to the high resolution of the preamplifiers but also to the very low bias current of the detector: $10 \mathrm{nA}$. A lot of efforts are being spent in improving the experimental setup. A new version of the

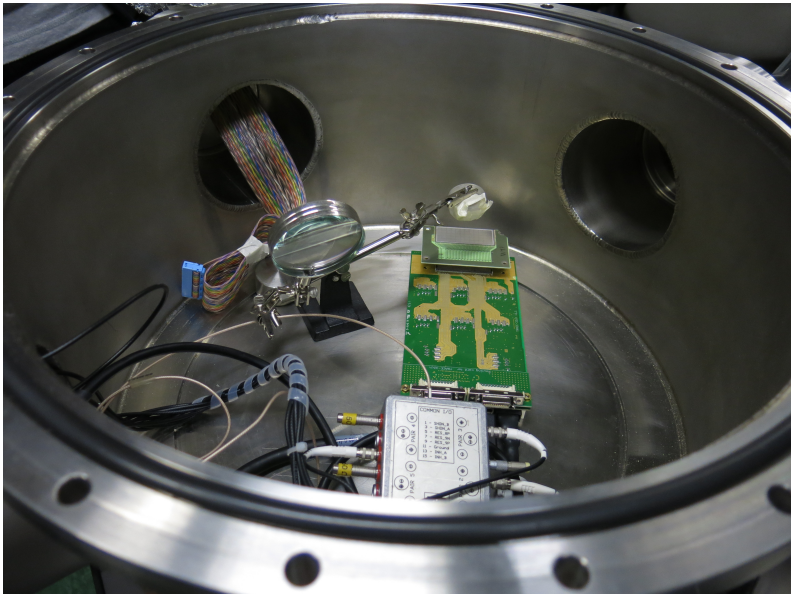

Fig. 3. Photo of the TRACE32ch v1.1 preamplifier board connected to a TRACE detector prototype inside a vacuum chamber with a triple ${ }^{241} \mathrm{Am}$ ${ }^{244} \mathrm{Cm}-{ }^{239} \mathrm{Pu}$ alpha source.

TABLE I

ABSOLUTE AND RELATIVE FWHM VALUES FOR THE FIVE MOST INTENSE PEAKS IN THE SPECTRUM OF THE ${ }^{241} \mathrm{AM}_{-}{ }^{244} \mathrm{CM}^{2}{ }^{239} \mathrm{PU}$ ALPHA SOURCE

\begin{tabular}{|c|c|c|}
\hline E [keV] & FWHM [keV] & FWHM \% \\
\hline 5155 & 23.81 & 0.46 \\
5443 & 26.28 & 0.48 \\
5486 & 21.56 & 0.39 \\
5763 & 24.80 & 0.43 \\
5805 & 22.02 & 0.38 \\
\hline
\end{tabular}

chip was designed and submitted to the foundry. It comprises eight channels specifically tailored for hole signals and one for electrons. The latter incorporates a new time-to-amplitude converter. In case of saturation this device is able to produce analog signals with amplitude proportional to the energy of the corresponding event. In this way the fast-reset device requires no more digital post-processing to boost the dynamic range of the preamplifier.

Future studies will address the issue of the external $1 \mathrm{G} \Omega$ resistor. Integrated solutions based on polysilicon resistors are being investigated [14], [15].

\section{ACKNOWLEDGMENT}

The authors would like to thank all the members of the GHT (GASPARD-HYDE-TRACE) European collaboration [16] [17] [18].

\section{REFERENCES}

[1] Mengoni, D. et al. Digital pulse-shape analysis with a trace early silicon prototype. Nuclear Instruments and Methods in Physics Research Section A: Accelerators, Spectrometers, Detectors and Associated Equipment 764, 241 - 246 (2014).

[2] Aliaga, R. et al. Conceptual design of the trace detector readout using a compact, dead time-less analog memory asic. Nuclear Instruments and Methods in Physics Research, Section A: Accelerators, Spectrometers, Detectors and Associated Equipment 800, 34-39 (2015).

[3] Aliaga, R. et al. Plas: A compact, self-triggered, dead time-less, high channel count analog memory asic for trace. 2015 IEEE Nuclear Science Symposium and Medical Imaging Conference, NSS/MIC 2015 (2015). 
[4] Capra, S. et al. Design of an integrated low-noise, low-power charge sensitive preamplifier for gamma and particle spectroscopy with solid state detectors. 2014 IEEE Nuclear Science Symposium and Medical Imaging Conference, NSS/MIC 2014 (2014).

[5] Capra, S., Mengoni, D., Aliaga, R., Gadea, A. \& Pullia, A. Experimental performance of the i2c integrated multichannel charge-sensitive preamplifier of trace. 2015 IEEE Nuclear Science Symposium and Medical Imaging Conference, NSS/MIC 2015 (2015).

[6] Pullia, A., Zocca, F. \& Capra, S. An integrated low-noise chargesensitive preamplifier with virtually unlimited spectroscopic dynamic range. IEEE Nuclear Science Symposium Conference Record 693-696 (2012).

[7] Pullia, A. \& Capra, S. Performance evaluation of an innovative integrated charge-sensitive preamplifier with dynamic range booster. 2015 IEEE Nuclear Science Symposium and Medical Imaging Conference, NSS/MIC 2015 (2015).

[8] Zocca, F., Pullia, A., Bazzacco, D. \& Pascovici, G. A time-overthreshold technique for wide dynamic range gamma-ray spectroscopy with the agata detector. Nuclear Science, IEEE Transactions on $\mathbf{5 6}$ 2384-2391 (2009).

[9] Pullia, A., Zocca, F., Pascovici, G. \& Bazzacco, D. Extending the dynamic range of nuclear pulse spectrometers. Review of Scientific Instruments 79 (2008).

[10] Zocca, F., Pullia, A., Bazzacco, D. \& Pascovici, G. Wide dynamic range front-end electronics for gamma spectroscopy with a hpge crystal of agata. IEEE Nuclear Science Symposium Conference Record 1, 7-13 (2007).

[11] Pullia, A. \& Zocca, F. Extending the dynamic range of a chargepreamplifier far beyond its saturation limit: A $0.35 \mathrm{um}$ cmos preamplifier for germanium detectors. IEEE Nuclear Science Symposium Conference Record 1919-1923 (2009).

[12] Pullia, A. et al. The agata charge-sensitive preamplifiers with builtin active-reset device and pulser. IEEE Nuclear Science Symposium Conference Record 3, 1411-1414 (2004). Cited By 4.

[13] Pullia, A., Boiano, C., Bassini, R. \& Pascovici, G. Active reset of digitized preamplifiers for ionizing-radiation sensors. IEEE Transactions on Nuclear Science 51, 831-835 (2004). Cited By 1.

[14] Capra, S. \& Pullia, A. Study of the effects of parasitic capacitance on large integrated feedback resistors for charge-sensitive preamplifiers. 2014 IEEE Nuclear Science Symposium and Medical Imaging Conference, NSS/MIC 2014 (2014).

[15] Pullia, A. \& Capra, S. Design of a resistorless asic preamplifier for hpge detectors with non-linear pole/zero cancellation and controlled fast-reset feature. IEEE Nuclear Science Symposium Conference Record 86-90 (2012).

[16] Beaumel, D. The GASPARD project. Nucl. Instrum. Meth. B317, 661663 (2013).

[17] Dalla Betta, G.F. et al. Hybrid detectors of neutrons based on 3d silicon sensors with polysiloxane converter. IEEE Nuclear Science Symposium Conference Record (2013).

[18] Spes: the hope of the legnaro national laboratory. URL https://web.infn. it/spes/index.php/research-on-nuclear-physics/150-trace. 